\title{
Listeners form average-based representations of individual voice identities - even when they have never heard the average
}

Nadine Lavan ${ }^{1,2}$, Sarah Knight ${ }^{1} \&$ Carolyn McGettigan ${ }^{1,2}$

${ }^{1}$ Department of Speech, Hearing and Phonetic Sciences, University College London

${ }^{2}$ Department of Psychology, Royal Holloway, University of London

Correspondence to:

Nadine Lavan, Department of Speech, Hearing and Phonetic Sciences, University College London, 2 Wakefield Street, London WC1N 1PF, United Kingdom. E-mail: n.lavan@ucl.ac.uk

or

Carolyn McGettigan, Department of Speech, Hearing and Phonetic Sciences, University College London, 2 Wakefield Street, London WC1N 1PF, United Kingdom. E-mail: c.mcgettigan@ucl.ac.uk

Acknowledgements: This work was supported by a Research Leadership Award from the Leverhulme Trust (RL-2016-013) awarded to Carolyn McGettigan 


\section{Abstract}

Models of voice perception propose that identities are encoded relative to an abstracted average or prototype. While there is some evidence for norm-based coding when learning to discriminate different voices, little is known about how the representation of a single voice identity is formed through variable exposure to that individual's voice. In two experiments, we show evidence that participants form abstracted representations of individual voice identities based on averages, despite having never been exposed to these averages during learning. We created 3 perceptually distinct voice identities, fully controlling their within-person variability. Listeners first learned to recognise these identities based on ring-shaped distributions located around the perimeter of within-identity voice spaces - crucially, these distributions were missing their centres. At test, listeners' accuracy for old/new judgements was higher for stimuli located on an untrained distribution nested around the centre of each ring-shaped distribution compared to stimuli on the trained ringshaped distribution.

\section{Introduction}

The question of how we learn and represent person identity has long been debated. For voices, a prominent view proposes that different identities are encoded on a multidimensional voice space in relation to a prototype voice. In such a view, the prototype is thought to be a representation of either an average voice, or a very frequently encountered voice (Andics, McQueen, Petersson, Gal, Rudas \& Vidnyanszky, 2010; Baumann \& Belin, 2010; Latinus, McAleer, Bestelmeyer \& Belin, 2013; Latinus \& Belin, 2011; Papcun, Kreiman \& Davies, 1989; see Maguinness, Roswandowitz \& von Kriegstein, 2018 and Mathias \& von Kriegstein, 2014 for 
reviews). The location of an individual voice relative to the prototype has perceptual implications: Voices that are further away from this between-person prototype are more perceptually distinctive (Mullennix, Ross, Smith, Kuykendall, Conard \& Barb, 2011; Latinus et al., 2013; Papcun et al., 1989; Valentine, 1991 for faces) and an identity's distance to the prototypical voice affects how well it is remembered and recognised. For example, some studies find evidence that distinctive voices are more reliably remembered (Mullenix et al., 2011; Yarmey, 1991), while others find evidence for the opposite pattern, with recognition remaining more reliable after a delay for prototypical voices (Papcun et al., 1989).

Studies exploring prototype- or norm-based coding tend to conceptualise different voice identities as single points in the voice space. They therefore focus on betweenperson variability, and on how listeners discriminate between separate identities. While this captures one important aspect of how we process voice identity information, it neglects the fact that individual voices are highly flexible. The acoustic and perceptual properties of a single person's voice can vary dramatically depending on the type of speech or vocalisations produced (shouting vs. reading aloud vs. joking with friends vs. whispering; Lavan, Burton, Scott \& McGettigan, 2018a). Listeners are therefore not only required to encode how different peoples' voices differ from each other; they also need to represent how the different instances of a single person's voice belong to the same identity. This can be a challenging task: it has been shown that listeners who are unfamiliar with a voice struggle to match, or "tell together", naturally-varying instances of speech produced by the same voice (Lavan, Burston \& Garrido, 2018b). Similarly, accurately perceiving identity across very different vocalisations (laughter vs. vowels) is challenging for familiar and unfamiliar voices 
alike (Lavan, Scott \& McGettigan, 2016). By failing to explicitly account for how withinperson variability impacts on the learning and perception of voices, current models of voice identity perception remain underspecified.

While norm-based coding and the extraction of summary statistics are at the centre of many models of identity perception, only a relatively small number of empirical studies has provided positive evidence of such a mechanism for faces and voices. In these studies, participants were presented with the voices or faces of familiar (or familiarised) identities: some of these stimuli were unmanipulated voice recordings/images of faces, while others were various averages derived from an increasing number of original stimuli. This approach assumes that the nature of a within-person representation is approximated by these averaged stimuli with the rationale that averaging preserves the diagnostic information about the person's identity while reducing non-diagnostic variability introduced by external factors (for voices: competing noises, quality of the signal transmitted, etc.) across different instances (Burton, Jenkins \& Schweinberger, 2011; Burton, Jenkins, Hancock, White, 2005; Fontaine, Love \& Latinus, 2017). A study of voice processing by Fontaine and colleagues (2017) explored how recognition accuracy and associated reaction times are affected by averaging single vowels produced by familiar or newly trained-tofamiliar voices. For familiar celebrity voices, the authors indeed find that the higher the number of recordings contributing to an average stimulus, the higher the explicit identity recognition accuracy, with reaction times correspondingly decreasing. The opposite effect is, however, reported for the trained-to-familiar voices: here, recognition accuracy decreased with increasing averageness, while reaction times remained stable. Why the effects did not replicate across the experiments is uncertain. 
More evidence for averageness aiding identity perception comes from a study using familiar (celebrity) faces as stimuli: here, reaction times decreased when the number of individual images used to create the average increased (Burton et al., 2005). Thus, averages may under certain circumstances form a meaningful part of how familiar voices (and faces) are represented.

Evidence for how an average may become a meaningful part of a representation can be found in studies of ensemble coding using sets of faces. It has been shown that viewers routinely extract summary statistics from sets of faces, such as the average emotional expression of faces (Haberman, Harp, \& Whitney, 2009; Haberman \& Whitney, 2007, 2009), and gender (Haberman \& Whitney, 2007). For identity processing, there is evidence that average identity information is represented for unfamiliar as well as familiar faces, and for sets of images of a single identity as well as multiple identities presented simultaneously or sequentially (de Fockert \& Wolfenstein, 2009; Neumann, Schweinberger \& Burton, 2013; Kramer, Ritchie \& Burton, 2015). Studies specifically probing within-identity processing in faces have shown that when viewers are briefly presented - simultaneously or sequentially - with a small number of variable pictures of a single identity, (previously unseen) average pictures derived from all presented pictures are labelled as previously seen with the same frequency as the individual pictures viewers have actually seen before (Kramer et al., 2015). Notably, viewers also seem to retain information about the specific exemplars viewed alongside these averages (Kramer et al., 2015; Neumann et al., 2013). In the auditory modality, there is evidence that listeners extract summary statistics from heard stimuli, such as the mean frequency of small sets of pure tones (Albrecht, Scholl, \& Chun, 2012; Piazza, Sweeny, Wessel, Silver \& Whitney, 2013) 
and sound textures (McDermott \& Simoncelli, 2011; McDermott, Schmetisch \& Simoncelli, 2013). To our knowledge, however, no study has directly probed whether summary statistics are extracted for voice identities.

The extraction of summary statistics from sets of stimuli seems to be commonplace in perception, but what is its purpose? Encoding abstracted statistical information compared to high-resolution and possibly redundant exemplar-based information is computationally efficient. The extraction of summary statistics has therefore been proposed as a candidate mechanism for how representations of individual face identities are formed (cf. Kramer et al., 2015). However, the studies on ensemble coding reviewed above rely on the brief presentation of a relatively small set of stimuli and do not entail any formal training or familiarisation procedure. It is thus unclear whether and how the extraction of summary statistics from faces using these paradigms extends to 1) voices and 2) paradigms that focus on training listeners to learn to recognise different voice identities and thus form representations of these voices.

We asked whether listeners extract summary statistics of salient acoustic cues from a distribution of voice samples when forming representations of individual voice identities. For this purpose, we created a two-dimensional acoustic voice space that enabled us to fully describe and control the variability in our stimuli and thus specify summary statistics. We created and perceptually validated artificial voice identities from an original voice by systematically manipulating two acoustic parameters that have been shown to be highly salient cues for the perception of voice identity (Baumann \& Belin, 2010; Kreiman \& Sidtis, 2011): glottal pulse rate (GPR), and 
apparent vocal tract length (VTL). These voice identities were therefore by definition matched on all properties apart from those we manipulated. In two experiments, listeners then learned to recognise these 3 perceptually distinct voice identities by being trained on ring-shaped distributions of GPR and VTL, with each distribution defining the perimeter of a nominal within-person voice space. Crucially, each of these ring-shaped distributions was missing its geometric centre (i.e. the average of the salient acoustic properties). At test, listeners provided old/new judgements for stimuli located on both the trained ring-shaped distribution and an untrained distribution nested around the centre of each ring-shaped distribution (i.e. covering the previously untrained average of the trained distribution). If acoustic averages are abstracted when forming representations of new individual voice identities, we predicted that accuracy on an old/new recognition task for learned voices should be the same or better for untrained stimuli located around the geometric average of the distribution than for the trained stimuli located on the ring-shaped distribution. Similarly, we predicted that accuracy should increase for stimuli that are acoustically closer to the centre or average. If averages are not abstracted, we predicted that accuracy on the old/new judgements task would be worse for the untrained stimuli in the centre of each within-identity voice space, compared to accuracy for trained stimuli located on the ring-shaped distribution. This should also be reflected in decreasing accuracy with increasing acoustic proximity to the geometric centre.

\section{Experiment 1}

\section{Participants}

44 participants were tested online using Gorilla (gorilla.sc/about; Anwyl-Irvine, Massonié, Flitton, Kirkham \& Evershed, 2018). Participants were recruited via Prolific 
(prolific.ac) and were reimbursed for their time. The study was approved by the ethics committee at Royal Holloway, University of London. All participants were aged between 18 and 40 years, were native speakers of English, had no reported hearing difficulties and had an approval rate over $90 \%$ on Prolific. No participant had taken part in any pilot or validation studies associated with this project. 4 participants were excluded from this data set: 1 participant failed to give the correct response for more than $20 \%$ of vigilance trials (see Methods) and 3 participants did not perform significantly better than chance $( \pm 95 \%$ confidence intervals) for the last 15 trials of Training 2 (see Methods). The final participant sample thus included 40 participants (mean age: 29.37 years, SD $=6.05$ years; 19 female). This sample size was determined to be adequate through a power analysis based on a small pilot study ( $N$ =15) using a similar paradigm.

\section{Materials and Procedure}

\section{Speakers}

We recorded two male speakers (Speaker 1 and Speaker 2) of Standard Southern British English producing 69 sentences from the Bamford-Kowal-Bench corpus (Bamford, Kowal, \& Bench, 1979; e.g. "The clown had a funny face"). Instead of reading out the original sentences, however, speakers were instructed to replace every original syllable with "na". These recordings formed the basis of the training and test voices used later. This type of "delexicalised" stimulus was chosen to avoid interference from lexical information in the stimuli, while retaining many other characteristics of the voice, such as pitch, intonation, speech rate, and general voice quality. Sentences were recorded in a sound-attenuated booth using a Røde NT1A microphone. All sentences were saved as WAV files, normed across sentences to 
match the median pitch of each speaker, and finally normed for RMS amplitude. Stimuli were converted into MP3 format for use on the online testing platform. In the study, one of the speakers was used as the basis for the training voice IDs while stimuli based on the second speaker were introduced at the test phase. We counterbalanced the assignment of speakers to training and test phases across participants.

\section{Creating distinct voice identities}

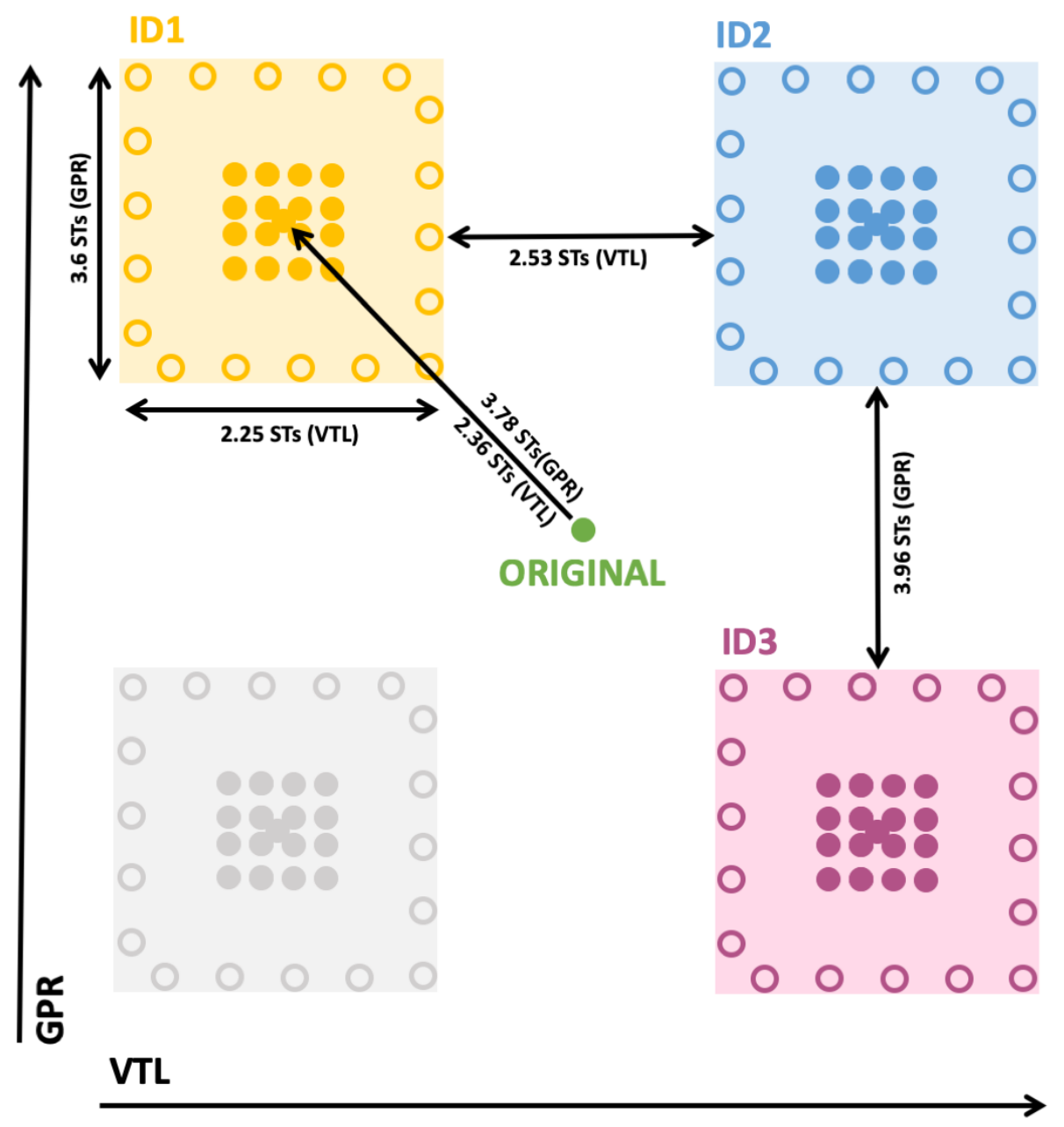

Figure 1 Illustration of the 2D voice space and the voice identities we created. VTL = vocal tract length, GPR = glottal pulse rate, ST = semitones. Shaded squares show the nominal within-person voice spaces for 4 identities created from a sentence produced by one original talker (green). Empty dots arranged around the perimeter of the individual voice spaces show the locations of stimuli from the ring-shaped distribution used during the learning phase; filled dots show the locations of the stimuli forming the centre distribution introduced at test. 
Based on each originally recorded speaker's sentences, we created 4 perceptually distinct voice identities ${ }^{1}$ by shifting the glottal pulse rate (GPR; related to the fundamental frequency and voice pitch perception) and vocal tract length (VTL; related to voice timbre perception) in Praat (Boersma \& Weenink, 2018) with the methods used by Darwin, Brungart and Simpson (2003), thus creating a two-dimensional voice space (Figure 1). GPR and VTL have previously been shown to be the most salient cues for identity perception (Baumann \& Belin, 2010; Gaudrain, Li, Ban \& Patterson, 2009; Holmes, Domingo \& Johnsrude, 2018; Kreiman \& Sidtis, 2011), allowing us to create perceptually distinct voice identities that differed only in two known acoustic properties (GPR and VTL). We were thus able to fully control, quantify and reproduce the properties and variability of the stimuli on which listeners were trained and tested. Furthermore, all stimuli were manipulated using the same procedure, thus minimising potential confounds introduced by comparing manipulated and unmanipulated stimuli.

In our voice space, changes in GPR and VTL were perceptually equated, where a 1 semitone change in VTL corresponded to a 1.6 semitone change in GPR (Gaudrain et al., 2009). The centres of each the 4 identities were displaced from the original voice by 3.78 semitones for GPR and 2.36 semitones for VTL. Around these centres, we created nominal within-person voice spaces to simulate some of the natural withinperson variability encountered in human voices (Figure 1; Lavan et al., 2018a). Each within-person voice space had a range of 2.25 semitones for VTL and 3.6 semitones for GPR; thus, stimuli furthest away from each other within each voice space was perceived somewhat ambiguous to unfamiliar listeners as to whether they could have

\footnotetext{
${ }^{1}$ Please note that "speaker" is consistently used throughout the paper to denote the originally recorded person, while "(voice) identity" is used for the discriminable identities used during the training and test sections of the task.
} 
been produced by the same speaker or not, as assessed via a speaker discrimination task (Supplementary Figure 1 and Gaudrain et al., 2009). After reviewing the perceptual qualities of the 4 voice identities, one voice (low GPR, short VTL; grey in Figure 1) was excluded due to this identity being perceived as sounding unnatural during perceptual piloting of the identities derived from one of the speakers, with stimuli being prone to distortions introduced by the acoustic manipulation. For information on the perceptual properties of the voice identities, please see Section 1 of the supplementary materials.

\section{$\underline{\text { Stimuli }}$}

For the main study, we created two distributions of locations from each identity's within-person voice space to allow us to test whether averages are formed when learning voice identities. The nature of these distributions is illustrated in Figure 1. For the learning phase of the study, where participants learned to recognise the 3 different identities, we created a ring-shaped distribution per identity (18 locations $\times 3$ identities). The stimuli included in these ring-shaped training distributions form a square around the perimeter of a nominal within-person voice space that crucially misses its centre. During learning, listeners were therefore never exposed to the average of the salient acoustic features for the individual identities. At test, we introduced a second type of distribution to participants (see Procedure). This type of distribution is nested within each ring-shaped training distribution and is located on and around the centre (and thus the average) of each nominal within-person voice space (17 locations $x 3$ identities). For the stimuli used in the main study, the different voice space locations were based on different underlying sentences (see Section 1 of the supplementary materials); sentences used at test had not been used in the training 
stimulus sets. Individual sentences were repeated 6 times each during training and 45 times during test. The different identities were created for both Speaker 1 and Speaker 2 - listeners were then trained on the identities created from one of the originally recorded speakers and tested on these identities plus the ones created for the other originally recorded speaker on an old/new judgement task (see Procedure). To minimise stimulus-specific effects, we furthermore created two stimulus sets. For the two sets of stimuli, the combinations of sentences and locations on the voice space were shuffled; for example: if Sentence 1 was manipulated to correspond to the centre location of the voice space of ID1 in Stimulus Set 1, this same sentence could be manipulated to correspond to a location on the ring-shaped distribution for ID1 in Stimulus Set 2. These stimulus sets were counterbalanced across participants.

\section{Procedure}

Participants first completed a headphones screening (Woods, Siegel, Traer \& McDermott, 2016) before completing two brief learning phases (Training 1 and Training 2). For Training 1, participants were presented with stimuli covering all 18 locations on the ring-shaped training distribution while a name (Peter, James or Michael) was presented on the screen. These presentations were blocked by identity and the order of identities was randomised across participants. Participants were instructed to listen attentively while memorising the different identities and their names. No responses were collected during this training phase. For Training 2, participants were presented with the same stimuli again in fully randomised order and were asked to complete a 3-way forced choice recognition task ("Is this Michael, James or Peter?") with audio-visual feedback on whether their response was correct or not. Both learning phases were self-timed and lasted on average between 5-8 
minutes in total. Performance for the final 15 trials of Training 2 were used as an index to track whether listeners had learned to recognise the two identities. These data showed listeners were able to correctly identify the 3 voice identities with high accuracy towards the end of the learning phase (mean accuracy $=88.6 \%, \mathrm{SD}=9.9 \%$; chance level $=33 \%$ ).

After this learning phase, participants completed an old/new judgement task for the test phase. Listeners were presented with 6 nominal identities: 3 learned identities based on one original speaker and 3 distractor identities based on the other original speaker. For listeners who had just learned identities based on Speaker 1, the distractor identities were based on Speaker 2, and vice versa. To increase the task difficulty, the GPR/F0 median was matched for the distractor identities to the GPR/F0 median for the learned identities. This was achieved by using the "change pitch" function in the Praat Vocal Toolkit (Corretge, 2012) to match the recordings of the original speaker used for the distractor identities to the median pitch of the original speaker used for the trained identities. The distractor identities were created afterwards. VTL was not explicitly aligned across speakers: VTL estimations based on 5 of the unmanipulated recordings showed that Speaker 1 and 2 were well-matched in their VTLs (Speaker 1: $15.4 \mathrm{~cm}$ Speaker 2: $15.6 \mathrm{~cm}$; for methods see Reby \& McComb, 2003). We determined that this difference of $.2 \mathrm{~cm}$ in VTL broadly corresponded to around 2 manipulation steps within our voice space (each being .23 semitones for VTL). We, however, note that these estimates should be treated with caution, since small differences in vowel quality and formant measurement errors may have influenced these VTL estimates across speakers. Thus the learned and distractor identities overlapped fully on the GPR dimension and only partially on the 
VTL dimension. This task included stimuli from both the trained ring-shaped distributions (18 locations $\times 3$ identities $\times 2$ speakers (learned, distractor)) and the centre-distribution of the 3 identities (17 locations $\times 3$ identities $\times 2$ speakers (learned, distractor)). Participants additionally completed 20 vigilance trials: here, a computergenerated voice instructed listeners to either respond with "old voice" or "new voice" (see exclusion criteria). The order of presentation was fully randomised across participants. The task was self-paced and participants were advised to complete the task in one sitting, without taking breaks. It took participants between 15 and 20 minutes to complete.

\section{Data availability}

Data from the main experiment and example stimuli have been deposited on the Open Science Framework: https://osf.io/xkbd6/

\section{Results}

\section{Learned identities: Confirmatory analyses}

To assess the relationship between accuracy and the location of stimuli in the voice space for the learned identities (i.e. not taking the data from the distractor identities into account), we ran a binomial intercept-only generalised linear mixed model (GLMM) using Ime4 (Bates, Maechler, Bolker \& Walker, 2014) in the $R$ environment ( $R$ Core Team, 2013). The model specification is detailed in the formula below. Note that we diverge from the preregistered analyses across all analyses reported in this paper in terms of the random effects structure, to avoid singular fits. 


$$
\begin{gathered}
\text { glmer(Accuracy Location + (1|participants)+(1|ID by speaker/stimulus } \\
\text { set/stimulus), family="binomial") }
\end{gathered}
$$

Statistical significance was established via likelihood ratio tests contrasting the full model including the fixed effect plus the random effects with a null model that did not include the fixed effect (Winter, 2013). Coefficients were transformed into probabilities (probability $=\exp [\mathrm{coeff}] /[1+\exp [\mathrm{coeff}])$ for ease of interpretation. These models confirmed that the type of distribution had an effect on accuracy (coefficient of -..32, $S E=.09$; probability $=.42$ ) and the comparison of the full and null model was significant $\left(X^{2}[1]=11.96, p=.001\right)$. Accuracy was higher for stimuli of the previously unheard centre distribution compared to stimuli from the trained, ring-shaped distribution (Figure 2a on the left). This trend was apparent for 5 out of 6 trained identities (Figure 2a on the right).

To assess whether there is a relationship between accuracy and acoustic distance to the centre of each identity's voice space, we ran a complementary binomial GLMM.

gImer(Accuracy $\sim 2 D$ Euclidean distance to the centre + (1|participants) $+(1 \mid I D$ by speaker/stimulus set/stimulus), family="binomial") 


\section{Learned identities}

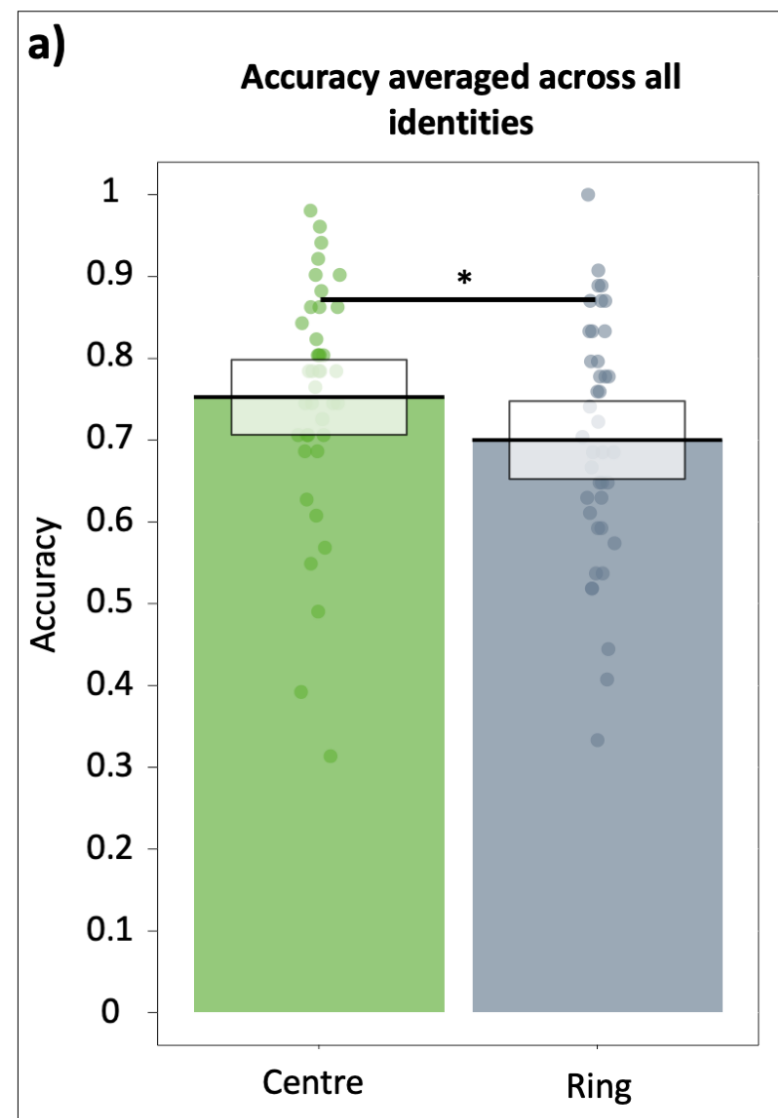

i)
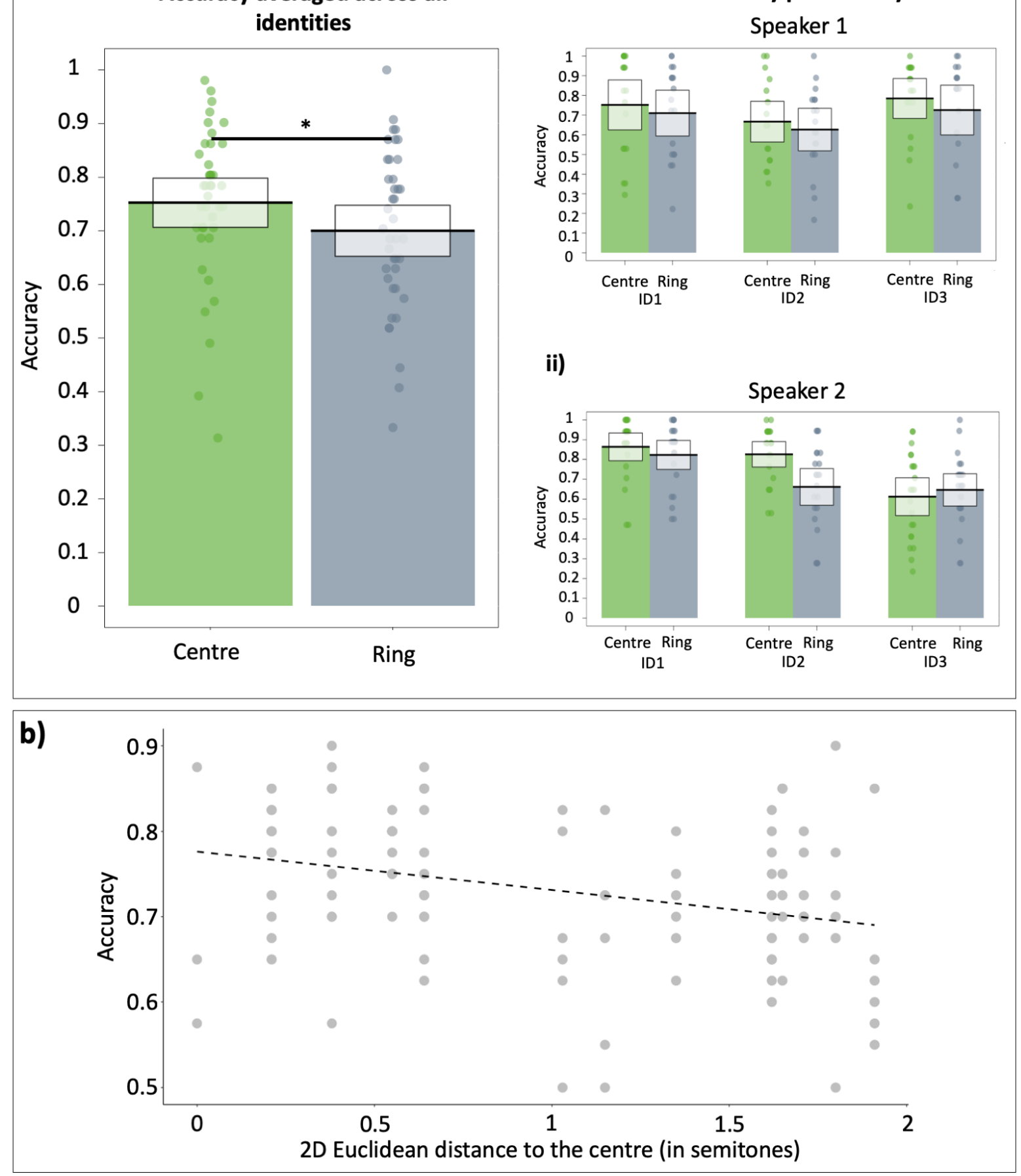

Figure 2 Summary of old/new recognition performance for learned identities for Experiment 1. a) Accuracy for the two distributions is plotted i) averaged across all identities and both speakers, and ii) broken down by identity and speaker. Boxes show the $95 \%$ confidence intervals, dots indicate the mean accuracy per participant. b) A scatterplot plotting each stimulus' 2D Euclidean distance in semitones to the centre of each identity's nominal voice space against the average performance for each location on the voice space. 


\section{Learned identities: Exploratory analyses}

We furthermore conducted exploratory analyses assessing whether the reported effects of acoustic distance to the centre hold for each dimension independently. Models were identical to the ones reported above with the distance in semitones for each dimension. For both GPR and VTL dimensions, accuracy increased the closer an item was to the centre of the training distribution. This effect was, however, only significant for VTL (VTL: coefficient $=-.41$, SE = .12; probability = .40; $x^{2}[1]=10.98$, $p=.001$, GPR: coefficient $=-.10, \mathrm{SE}=.08 ;$ probability $\left.=.48 ; x^{2}[1]=1.73, p=.189\right)$.

\section{Distractor identities: Exploratory analyses}

To assess the relationship between accuracy and the location in the space (ringshaped versus centre) for the distractor identities, we ran a GLMM analysis identical in structure to the one run for the learned identities. These analyses confirmed that the location on the voice space had an effect on accuracy (coefficient of $.52, S E=.08$, probability $\left.=.63 ; X^{2}[1]=40.00, p<.001\right)$. Intriguingly, we observed the opposite trend to that for learned identities (Figure 3a): Accuracy was higher for stimuli on the ringshaped distribution and lower for the centre distribution, a trend that holds for all 6 identities.

We further explored whether there is a relationship between accuracy and acoustic distance to the centre of each identity's voice space, again mirroring the GLMMs specified for the learned identities.

glmer(Accuracy $\sim 2 D$ Euclidean distance to the centre $+(1 \mid$ participants $)+(1 \mid I D$ by speaker/stimulus set/stimulus), family="binomial") 


\section{Distractor identities}

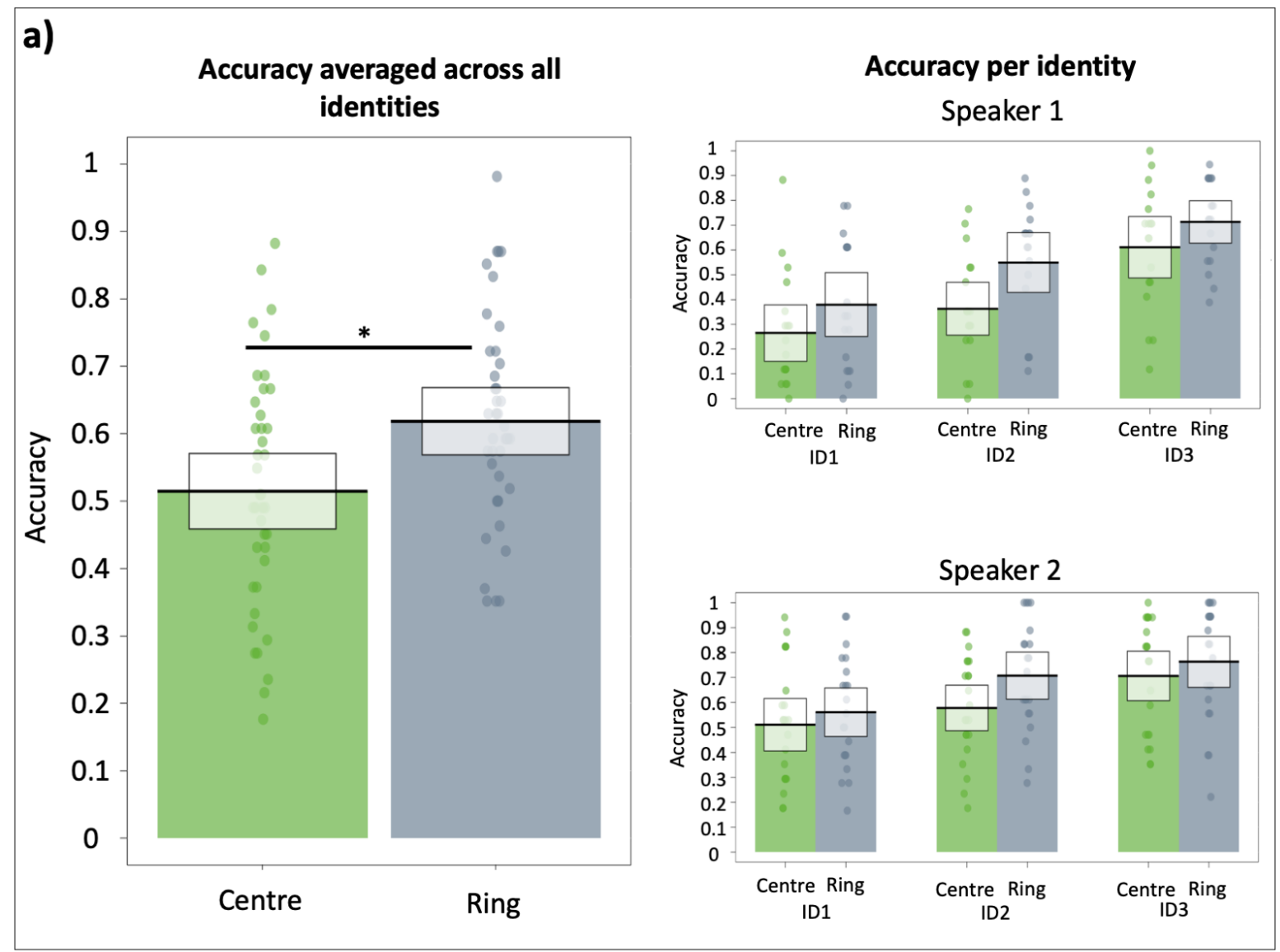

\section{b)}

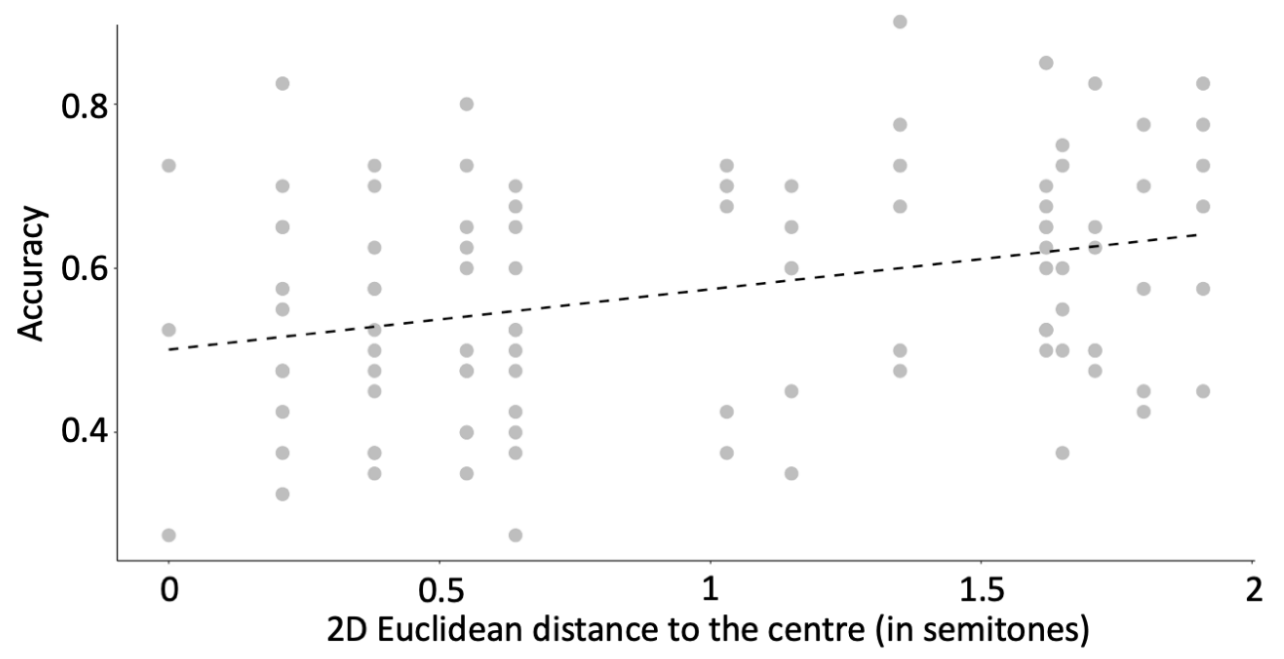

Figure 3: Summary of old/new recognition performance for distractor identities for Experiment 1. a) Accuracy for the two distributions is plotted i) averaged across all identities and both speakers and ii) broken down by identity and speaker. Boxes show the $95 \%$ confidence intervals, dots indicate the mean accuracy per participant. b) A scatterplot plotting the 2D Euclidean distance in semitones to the centre of each identity's nominal space against the average performance, for each location on the space. 
These models also showed the opposite effect to what we observed for the learned identities: There was a positive relationship between accuracy and distance to the centre $\left(\right.$ coefficient of $.45, S E=.07 ;$ probability $\left.=.61 ; X^{2}[1]=43.783, p<.001\right)$. Accuracy thus decreases the closer a stimulus is to the centre of the trained ringshaped distribution, or rather, the further it is from the location of the trained stimuli (see Figure 3b). This effect is also true when considering VTL and GPR separately

$\left(\mathrm{VTL}\right.$ : coefficient of $.58, S E=.11 ;$ probability $=.64 ; x^{2}[1]=26.71, p<.001 ;$ GPR: coefficient of $.39, S E=.07 ;$ probability $\left.=.60 ; X^{2}[1]=31.03, p<.001\right)$.

\section{Discussion}

The experiment suggests that averages are indeed abstracted during the learning of novel voice identities: accuracy was higher for the previously untrained centre of the training distribution than the training distribution itself. This effect was also apparent when modelling the location on the within-person voice spaces in terms of the acoustic distance between a stimulus' acoustic properties and the centre of an identity's voice space: accuracy increased for stimuli closer to the centre. Intriguingly, the opposite pattern of results was found in an exploratory analysis of the distractor identities.

\section{Experiment 2}

In Experiment 2, we intended to replicate and extend our findings. In this follow-up experiment we thus used the same experimental design and test stimuli, but now trained listeners on independent recordings of full sentences with their linguistic content intact, instead of using "delexicalized" sentences. Our predictions were identical to those stated for Experiment 1. Replicating our effect in this experiment would show that the extraction of averages generalises to more naturalistic stimuli (by 
introducing linguistic content), even when listeners are required to transfer information about the learned identities across different types of stimuli from training to test, which is known to impair performance (see Lavan et al., 2018a for a review).

\section{Participants}

56 participants were tested online using Gorilla (gorilla.sc). Participants were recruited via Prolific (prolific.ac) and were reimbursed for their time. The study was approved by the local ethics committee. All participants were aged between 18 and 40 years, were native speakers of English, had no reported hearing difficulties and had an approval rate over $90 \%$ on Prolific. No participant had taken part in any pilot, validation studies associated with this project, or Experiment 1. Six participants were excluded from the sample as they did not perform significantly better than chance $( \pm 95 \%$ confidence intervals) for the last 15 trials of Training 2. The final participant sample thus included 50 participants (mean age: 29.57 years, SD = 6.39 years; 32 female, 1 other).

\section{Materials and Procedure}

\section{Speakers and stimuli}

Stimuli were produced by the same two speakers as in Experiment 1. Stimuli included 54 sentences from the Bamford-Kowal-Bench corpus (17 items x 3 identities; e.g. "They're buying some bread", "Children like strawberries”; Bench, Kowal \& Bamford, 1979) that formed the basis for both learning phases (Training 1 and 2). No sentence was repeated within each training phase. The stimuli for the test phase remained the same as in Experiment 1.

\section{Procedure}

The procedure was identical to the one reported in Experiment 1 


\section{Data availability}

Data from the main experiment have been deposited on the Open Science Framework: https://osf.io/xkbd6/

\section{$\underline{\text { Results }}$}

\section{Learned identities: Confirmatory analyses}

Data were analysed in the same way as in Experiment 1: We first contrasted accuracy for the centre distribution versus the ring-shaped distribution, for the learned identities only. These models confirmed that the type of distribution had an effect on accuracy (coefficient of $-.35, S E=.07 ;$ probability $=.41 ; X^{2}[1]=25.34, p<.001$ ). We thus replicate the effect reported in Experiment 1 showing that accuracy is higher for stimuli from the previously unheard centre distribution compared to stimuli from the trained, ring-shaped distribution (Figure 4a on the left). This trend was apparent for all 6 trained identities (Figure 4a, right).

We then tested whether accuracy was related to the acoustic distance to the centre. These models confirmed that there was a negative relationship between the two measures (coefficient of $-.25, S E=.06$; probability $\left.=.44 ; X^{2}[1]=19.845, p<.001\right)$. Thus, we replicate our findings from Experiment 1, showing that accuracy increases the closer a stimulus is to the centre of the trained distribution (Figure $4 b$ ). 


\section{Learned identities}

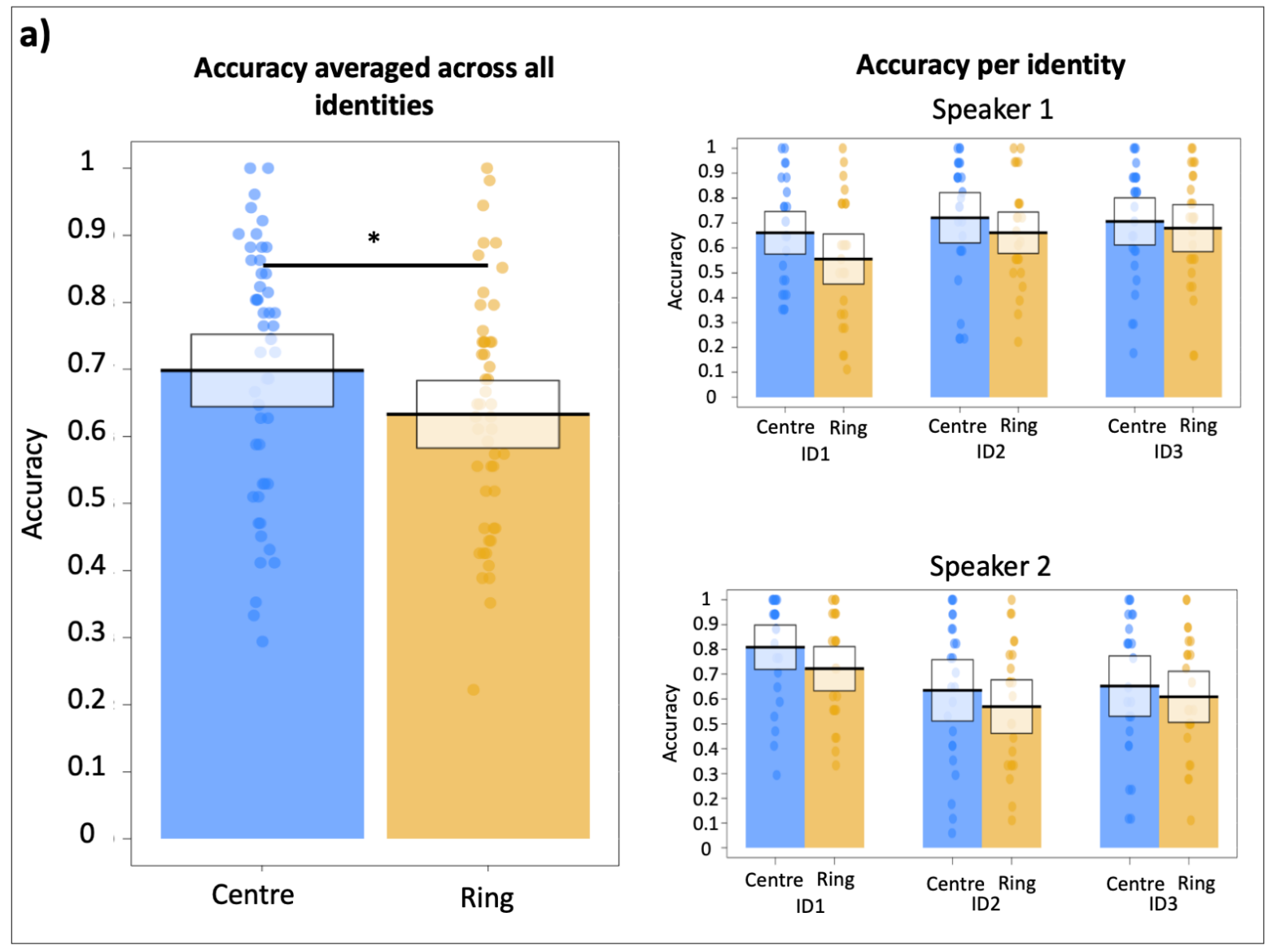

b)

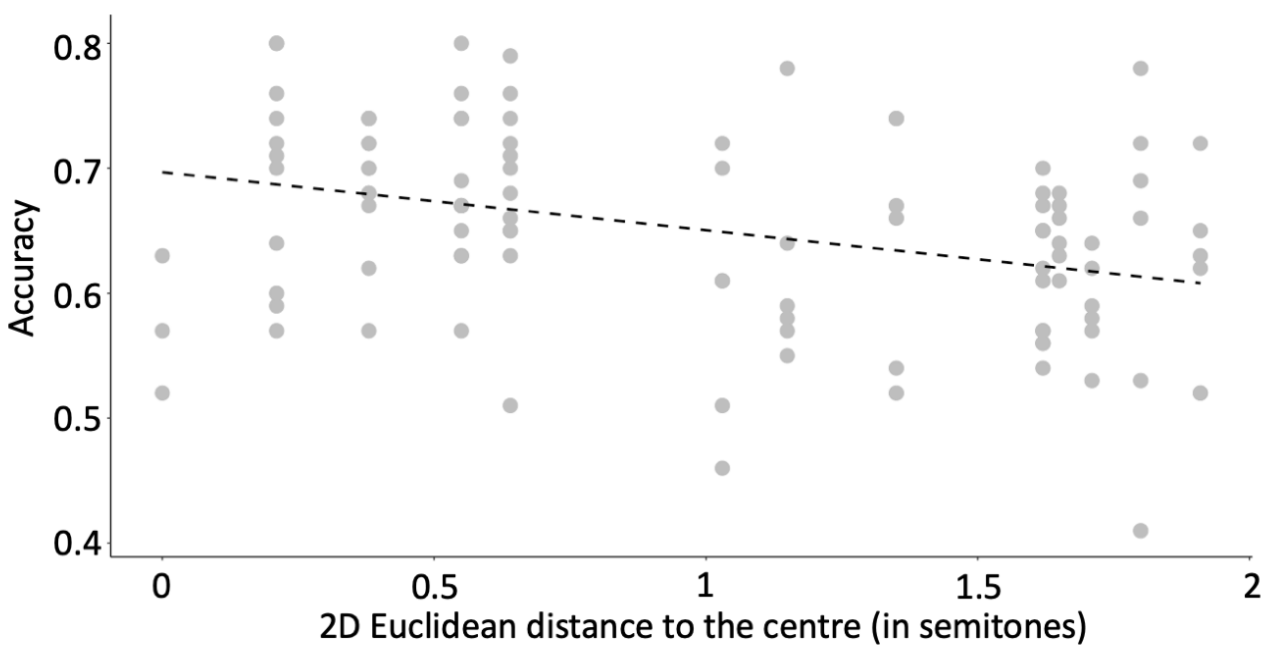

Figure 4 Summary of old/new recognition performance for learned identities for Experiment 2. a) Accuracy for the two distributions is plotted i) averaged across all identities and both speakers, and ii) broken down by identity and speaker. Boxes show the $95 \%$ confidence intervals, dots indicate the mean accuracy per participant. b) A scatterplot plotting each stimulus' 2D Euclidean distance in semitones to the centre of each identity's nominal voice space against the average performance for each location on the voice space. 


\section{Learned identities: Exploratory analyses}

We again assessed whether the effect of acoustic distance holds for both GPR and VTL dimensions individually. Models were identical to the ones reported for Experiment 1 . We replicate our findings showing that accuracy increased the closer an item was to the centre of the training distribution for each of the dimensions (VTL: coefficient $=-.34, S E=.09 ;$ probability $=.42 ; X^{2}[1]=13.40, p<.001$, GPR: coefficient $=-.20, S E=.06 ;$ probability $\left.=.45 ; X^{2}[1]=11.77, p<.001\right)$.

\section{Distractor identities: Exploratory analyses}

We also conducted the analyses for the data from the distractor identities as detailed above for Experiment 1. In the model contrasting accuracy for the centre distribution versus the ring-shaped distribution, we again find that the location on the voice space has an effect on accuracy (coefficient of $.20, S E=.06 ;$ probability $=.55 ; X^{2}[1]=9.75$, $p=.001$ see Figure 5a). The trend of better performance for the ring-shaped distribution holds for all 6 identities. Similarly, we replicate the finding showing an effect of acoustic distance to the centre on accuracy when considering distance in two dimensions (coefficient of .19, SE $=.05 ; X^{2}[1]=12.60, p<.001$, see Figure $5 b$ ) and also when considering each dimension individually (VTL: coefficient of .32, SE = .09; probability $=.55 ; X^{2}[1]=13.71, p<.001 ;$ GPR: coefficient of .15, SE $=.05$; probability $\left.=.54 ; X^{2}[1]=8.02, p=.005\right)$. 


\section{Distractor identities}
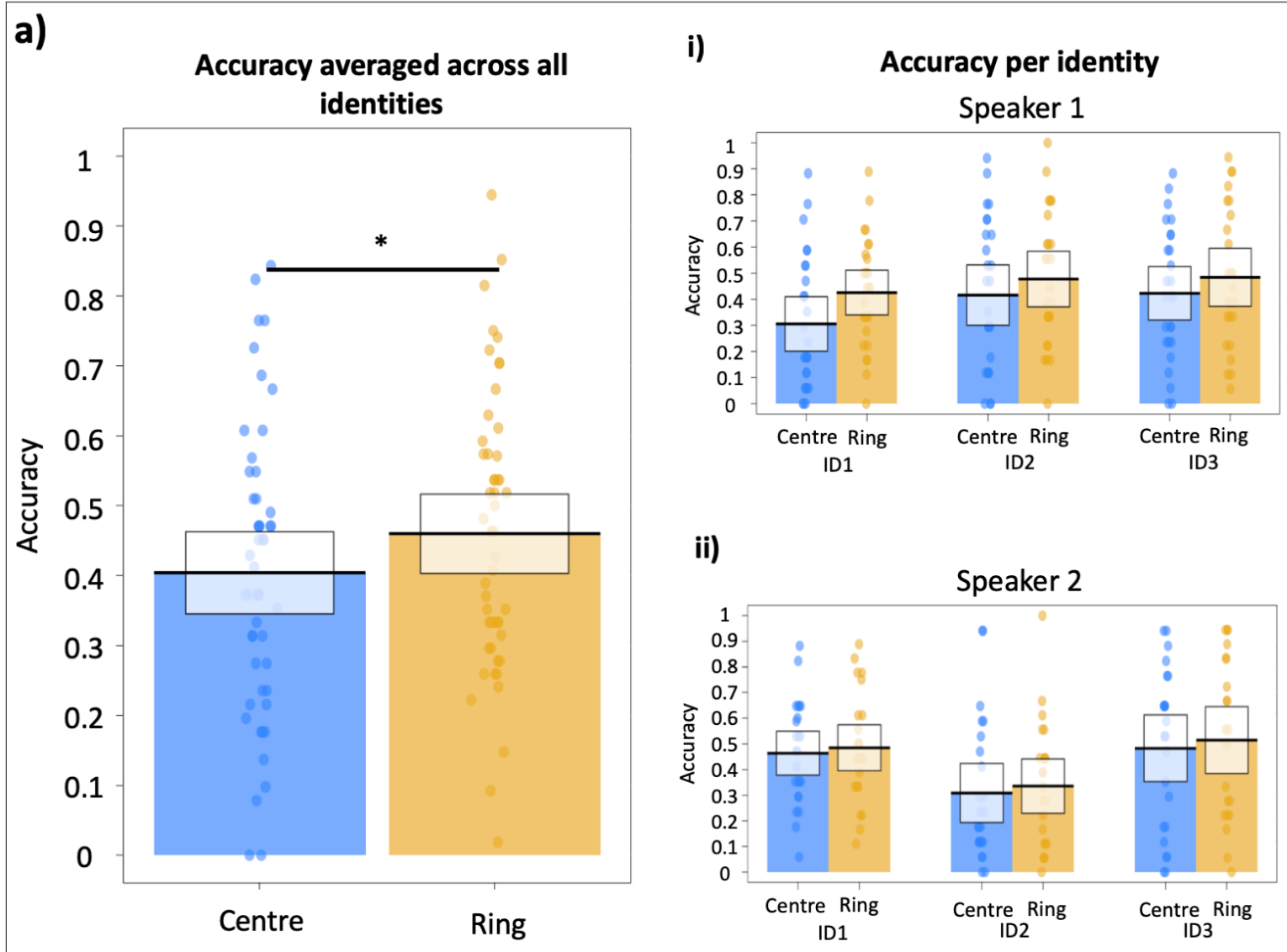

ii)

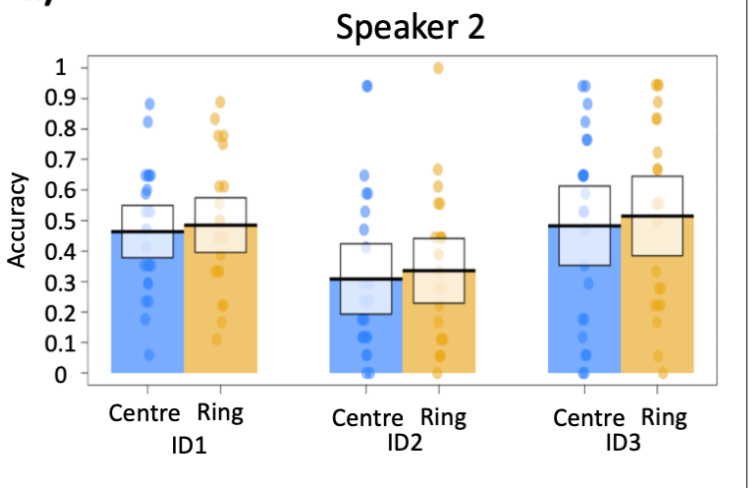

b)

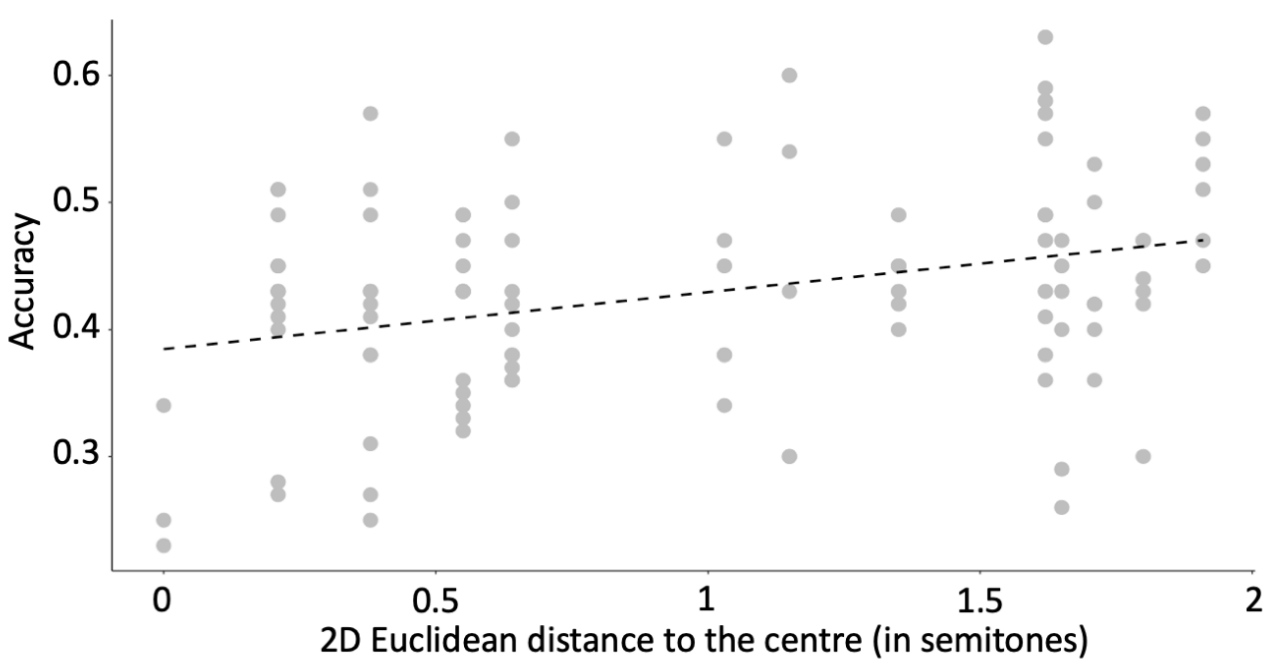

Figure 5: Summary of old/new recognition performance for distractor identities for Experiment 1. a) Accuracy for the two distributions is plotted i) averaged across all identities and both speakers and ii) broken down by identity and speaker. Boxes show the $95 \%$ confidence intervals, dots indicate the mean accuracy per participant. b) A scatterplot plotting the 2D Euclidean distance in semitones to the centre of each identity's nominal space against the average performance, for each location on the space. 


\section{Discussion}

In Experiment 2, we replicate our findings from Experiment 1, showing that listeners automatically abstract averages when learning new voice identities. Experiment 2 presents two key advances: First, the full sentence stimuli are more natural than the "delexicalized" sentences used in Experiment 1; thus we can show that averages are extracted from more naturalistic speech stimuli. Second, by introducing a difference between the nature of the training and the test stimuli, we can now show that the effect of averaging is not limited to the type of stimulus on which listeners are trained, but that it generalises across different types of stimuli. Thus, even though listeners in Experiment 2 were exposed to all the richness of the speakers' accent and idiosyncratic pronunciations - and were free to attend to these cues during training they nonetheless abstracted the average of the manipulated dimensions alongside this rich information and were able to generalise it to delexicalised test stimuli that were missing most of the pronunciation-related cues. In comparison to Experiment 1 , overall performance was lower (e.g. $69 \%$ for the centre distribution vs. $76 \%$ in Experiment 1): this is most likely an effect reflecting the cost of additional generalisation across stimulus types from training (sentences including lexical content) to test (delexicalized sentences).

\section{General discussion}

In the current experiments, we investigated whether individual voice averages are abstracted during the learning of new identities. Across two experiments, participants learned to recognise 3 new identities based on variable voice stimuli forming ringshaped distributions of stimuli located on a 2D voice space. Subsequent accuracy for an old/new recognition task was higher for an untrained distribution of stimuli grouped 
around the centre - the acoustic average - of the learned distribution than for stimuli located on the previously trained ring-shaped distribution. Since our participants were not given any specific instructions on how to learn the different identities, it is likely that the extraction of summary statistics occurred automatically and involuntarily during learning. Previous research has indeed shown that listeners automatically learn about the properties of artificially created complex sounds that vary systematically along two dimensions (Stilp, Rogers \& Kluender, 2010). All effects hold across two independent participant samples and two types of training stimulus: in Experiment 1, delexicalized sentences were used while in Experiment 2 we used sentences that included full linguistic content.

Models of voice perception have proposed norm-based coding for voice identities on a multidimensional voice space, highlighting average voices as perceptually meaningful prototypes in relation to which different identities are encoded. In the context of the extensive within-person variability of human voices, it is reasonable to assume that a similar process is at work when forming a representation of a new voice identity based on variable signals (Lavan et al., 2018a). The current studies are the first empirical demonstrations that participants indeed extract averages when forming abstracted representations of voice identities, despite having never been exposed to these averages during learning. These findings can thus be interpreted as preliminary evidence for the existence of abstracted, norm-based within-person representations of voice identity as a result of being exposed to a variable signal. Our results additionally show evidence against exemplar-based models of identity coding, which are frequently used as an alternative to norm-based coding models (e.g. Valentine, 1991 for faces). Exemplar-based models would predict the highest accuracy for stimuli 
that are acoustically closer to what was previously heard. Although we note that we define exemplars as specific locations in a voice space, as opposed to specific stimuli (i.e. we did not repeat the same recordings across learning and test), an exemplarbased account should still predict weaker test performance for previously unheard locations in the centre distribution. Since listeners were never exposed to the centre of the within-person voice spaces at training, they cannot have stored an exemplarbased representation for stimuli falling into this voice space. They may, however, have retained representations of exemplars from the trained ring-shaped distribution, to which listeners were previously exposed. Thus, an exemplar model of representation would have predicted the opposite to the observed pattern of results.

As part of exploratory analyses, we found that in both experiments, accuracy for the distractor identities exhibited the opposite pattern of results to the learned identities: accuracy (i.e. correctly labelling a distractor as "new") was higher for stimuli located on the ring-shaped distribution compared to stimuli located on the centre distribution. Similarly, accuracy now decreased the closer stimuli were to the centre (whether considering both acoustic properties together, or modelling them individually). How these exploratory findings should be interpreted remains unclear: We have, for example, little insight into how the manipulated acoustic cues may have shaped listeners' responses to the previously unheard distractor identities. For further speculative interpretations of these findings in relation to possible task-related effects or as further evidence for average-based coding, please see supplementary materials (Section 2). 
How can the current findings be integrated into existing models of voice perception? Given the flexibility and natural variability of our voices, we are likely to not only encode abstracted representations of how different voices relate to a single underlying prototype; we also need to form representations of how different variable exemplars of the same voice go together - that is, we must form a within-person representation. For the current studies we report that listeners extract identity-specific summary statistics when learning new voice identities. We propose that this process may underpin how representations of voice identities are formed (see also Kramer et al., 2015 for faces). If voice identity perception thus involves both within- and acrossidentity representations, the question arises of how these two representations or processes interact. First, there appears to be a logical hierarchy to the order in which within- and between-person representations are formed when learning a new voice. Upon first exposure to a new voice identity, it can be rapidly established where this identity falls on the between-person voice space (Baumann \& Belin, 2010): this voice space is already populated with a large number of familiar voices that we have encoded throughout our lives. These familiar voices have thus already mapped out and defined the dimensions and boundaries of the between-person voice space and have crucially created a prototypical voice that is used an anchor for between-person identity coding (Maguinness et al., 2018 for a review). Being exposed to a single exemplar may thus be sufficient to quickly assess with reasonable accuracy that the voice just heard is an unknown one. This hypothesis is supported by the finding that listeners who are unfamiliar with a set of voices have little trouble telling these voices apart with high accuracy, with performance comparable to that of listeners who are already familiar with the voices (Lavan et al., 2018b). Note, however, that false alarms (i.e. labelling unfamiliar voices as familiar) have also been reported (Hanley, Smith \& 
Hadfield, 1998). When first encountering a new voice, by definition no within-person representation or prototype exists until multiple exemplars have been heard. Thus, after establishing that a voice is indeed unknown, a within-person representation and voice space must be mapped out. Our results suggest that this may take place through extracting summary statistics that will eventually, through increasing exposure to this voice, form a stable within-person prototype (Maguinness et al., 2018).

The current studies have implemented a novel method to create distinctive voice identities featuring within-person variability. Tight control over the acoustic and perceptual properties of the stimuli was essential to allow us to create distributions of stimuli with known properties and quantify their statistical averages. Through this process, ecological validity was sacrificed: we introduced variability for each identity by changing two perceptually salient acoustic properties of the stimuli (GPR and VTL). While GPR changes significantly in everyday voice use, there are no good estimates of whether and how VTL might change (Gaudrain et al., 2009; Holmes et al., 2018). Natural variability of a voice is not two-dimensional but highly multi-dimensional, such that any number of acoustic features will be modified by simple changes in speaking style (conversational speech vs. read speech) or interlocutor (infant-directed speech vs. speaking with an adult; Lavan et al., 2018a). One promising method that can introduce higher-dimensional manipulations of variability may, for example, by morphing techniques through which continua between two voices can be created (Latinus \& Belin, 2011). It has furthermore been demonstrated that within-person variability may be idiosyncratic, that is, specific to an individual and therefore potentially diagnostic when perceiving identity as opposed to being noise (Burton, Kramer, Ritchie \& Jenkins, 2016 for faces): the identities created in the current study 
varied in terms of the absolute GPR and VTL, but how identities varied was identical. Finally, the variability of specific acoustic properties in everyday vocal behaviour is likely to follow a broadly normal distribution (Kinoshita, Ishihara \& Rose [2009] for GPR), marking our training distribution as highly artificial. This type of training distribution was, however, specifically chosen to avoid the confounding effects of frequency of exposure: for normally distributed variability, the most average samples will also be the ones that are heard most frequently (Valentine, 1991 for a discussion for faces).

The current study thus opens up exciting new avenues for future research to further elucidate the mechanisms underpinning the reported effects. For example, are listeners forming pure mathematical averages, or do they take the precise distribution of exemplars into account to form weighted-average, "prototypical" representations? This could be tested explicitly by examining how outlier exemplars influence the properties of learned averages. Further, is information about the variability also abstracted and/or encoded when forming representations? It will furthermore be a challenge to determine whether the averaging mechanisms suggested by our current data are comparable to the ones at work in richer, more naturalistic learning environments and over longer periods of exposure and learning: how much exposure is needed to observe these effects, and does this depend on the degree of withinperson variability encoded during training? Since we can see significant averaging effects already after a short retention span during which exemplar-based memory traces should still be available, we might predict that the average-based abstraction becomes yet stronger over longer retention periods during which representation of specific exemplars might progressively fade. Finally, the extraction of summary 
statistics can be observed in the visual as well as in the auditory modality, both in the context of ensemble coding as a result of being exposed to sets of stimuli for a very short amount of time and - as this study has also shown - in the context of longer learning or training paradigms: whether these findings rely on the same underlying mechanisms across modalities, or are unrelated, remains an open question. Ours is, however, a first promising investigation into the mechanisms used to form withinperson representation of voices, and highlights once again the importance of accounting for within-person variability in models of voice perception.

\section{References}

Albrecht, A. R., Scholl, B. J., \& Chun, M. M. (2012). Perceptual averaging by eye and ear: Computing summary statistics from multimodal stimuli. Attention, Perception, \& Psychophysics, 74(5), 810-815.

Andics, A., McQueen, J. M., Petersson, K. M., Gál, V., Rudas, G., \& Vidnyánszky, Z. (2010). Neural mechanisms for voice recognition. Neuroimage, 52(4), 1528-1540.

Anwyl-Irvine, A., Massonnié, J., Flitton, A., Kirkham, N., \& Evershed, J. (2018). Gorillas in our Midst: Gorilla. sc, a new web-based Experiment Builder. bioRxiv, 438242.

Bates, D., Maechler, M., Bolker, B., \& Walker, S. (2014). Ime4: Linear mixed-effects models using Eigen and S4. R package version, 1(7), 1-23.

Baumann, O., \& Belin, P. (2010). Perceptual scaling of voice identity: common dimensions for different vowels and speakers. Psychological Research 74(1), 110120.

Bench, J., Kowal, Å., \& Bamford, J. (1979). The BKB (Bamford-Kowal-Bench) sentence lists for partially-hearing children. British journal of audiology, 13(3), 108112.

Boersma, Paul \& Weenink, David (2018). Praat: doing phonetics by computer [Computer program].

Burton, A. M., Jenkins, R., \& Schweinberger, S. R. (2011). Mental representations of familiar faces. British Journal of Psychology, 102(4), 943-958.

Burton, A. M., Jenkins, R., Hancock, P. J., \& White, D. (2005). Robust representations for face recognition: The power of averages. Cognitive psychology, 51(3), 256-284. 
Burton, A. M., Kramer, R. S., Ritchie, K. L., \& Jenkins, R. (2016). Identity from variation: Representations of faces derived from multiple instances. Cognitive Science, 40(1), 202-223.

Corretge, Ramon (2012). Praat Vocal Toolkit. http://www.praatvocaltoolkit.com

Darwin, C. J., Brungart, D. S., \& Simpson, B. D. (2003). Effects of fundamental frequency and vocal-tract length changes on attention to one of two simultaneous talkers. The Journal of the Acoustical Society of America, 114(5), 2913-2922.

de Fockert, J., \& Wolfenstein, C. (2009). Rapid extraction of mean identity from sets of faces. The Quarterly Journal of Experimental Psychology, 62(9), 1716-1722.

Fontaine, M., Love, S. A., \& Latinus, M. (2017). Familiarity and voice representation: from acoustic-based representation to voice averages. Frontiers in psychology, 8, 1180.

Gaudrain, E., Li, S., Ban, V. S., \& Patterson, R. D. (2009). The role of glottal pulse rate and vocal tract length in the perception of speaker identity. In Tenth Annual Conference of the International Speech Communication Association.

Haberman, J., \& Whitney, D. (2007). Rapid extraction of mean emotion and gender from sets of faces. Current Biology, 17(17), 751-753.

Haberman, J., \& Whitney, D. (2009). Seeing the mean: ensemble coding for sets of faces. Journal of Experimental Psychology: Human Perception and Performance, 35(3), 718-734.

Haberman, J., Harp, T., \& Whitney, D. (2009). Averaging facial expression over time. Journal of vision, 9(11), 1.

Holmes, E., Domingo, Y., \& Johnsrude, I. S. (2018). Familiar Voices Are More Intelligible, Even if They Are Not Recognized as Familiar. Psychological science, 0956797618779083.

Kinoshita, Y., Ishihara, S., \& Rose, P. (2009). Exploring the discriminatory potential of F0 distribution parameters in traditional forensic speaker recognition. International Journal of Speech, Language \& the Law, 16(1), 91-111.

Kramer, R. S., Ritchie, K. L., \& Burton, A. M. (2015). Viewers extract the mean from images of the same person: A route to face learning. Journal of Vision, 15(4), 1-1.

Kreiman, J., \& Sidtis, D. (2011). Foundations of voice studies: An interdisciplinary approach to voice production and perception. John Wiley \& Sons.

Latinus, M., \& Belin, P. (2011). Anti-voice adaptation suggests prototype-based coding of voice identity. Frontiers in psychology, 2, 175.

Latinus, M., McAleer, P., Bestelmeyer, P. E., \& Belin, P. (2013). Norm-based coding of voice identity in human auditory cortex. Current Biology, 23(12), 1075-1080. 
Lavan, N., Burston, L. F., \& Garrido, L. (2018b). How many voices did you hear? Natural variability disrupts identity perception from unfamiliar voices. British Journal of Psychology.

Lavan, N., Burton, A. M., Scott, S. K., \& McGettigan, C. (2018a). Flexible voices: identity perception from variable vocal signals. Psychonomic Bulletin \& Review

Lavan, N., Scott, S. K., \& McGettigan, C. (2016). Impaired generalization of speaker identity in the perception of familiar and unfamiliar voices. Journal of Experimental Psychology: General, 145(12), 1604-1614.

Maguinness, C., Roswandowitz, C., \& von Kriegstein, K. (2018). Understanding the mechanisms of familiar voice-identity recognition in the human brain. Neuropsychologia.

Mathias, S. R., \& von Kriegstein, K. (2014). How do we recognise who is speaking. Frontiers in Biosciences (6), 92-109.

McDermott, J. H., Schemitsch, M., \& Simoncelli, E. P. (2013). Summary statistics in auditory perception. Nature neuroscience, 16(4), 493-498.

McDermott, J. H., \& Simoncelli, E. P. (2011). Sound texture perception via statistics of the auditory periphery: evidence from sound synthesis. Neuron, 71(5), 926-940.

Mullennix, J. W., Ross, A., Smith, C., Kuykendall, K., Conard, J., \& Barb, S. (2011). Typicality effects on memory for voice: Implications for earwitness testimony. Applied Cognitive Psychology, 25(1), 29-34.

Murphy, J., Ipser, A., Gaigg, S. B., \& Cook, R. (2015). Exemplar variance supports robust learning of facial identity. Journal of Experimental Psychology: Human Perception and Performance, 41(3), 577-581.

Neumann, M. F., Schweinberger, S. R., \& Burton, A. M. (2013). Viewers extract mean and individual identity from sets of famous faces. Cognition, 128(1), 56-63.

Papcun, G., Kreiman, J., \& Davis, A. (1989). Long-term memory for unfamiliar voices. The Journal of the Acoustical Society of America, 85(2), 913-925.

Piazza, E. A., Sweeny, T. D., Wessel, D., Silver, M. A., \& Whitney, D. (2013). Humans use summary statistics to perceive auditory sequences. Psychological science, 24(8), 1389-1397.

Reby, D., \& McComb, K. (2003). Vocal communication and reproduction in deer. Advances in the Study of Behavior, 33, 231-264.

Stilp, C. E., Rogers, T. T., \& Kluender, K. R. (2010). Rapid efficient coding of correlated complex acoustic properties. Proceedings of the national academy of sciences, 201009020.

Team, R. C. (2013). R: A language and environment for statistical computing. 
Valentine, T. (1991). A unified account of the effects of distinctiveness, inversion, and race in face recognition. The Quarterly Journal of Experimental Psychology Section A, 43(2), 161-204.

Winter, B. (2013). A very basic tutorial for performing linear mixed effects analyses. arXiv preprint arXiv:1308.5499.

Woods, K. J., Siegel, M. H., Traer, J., \& McDermott, J. H. (2017). Headphone screening to facilitate web-based auditory experiments. Attention, Perception, \& Psychophysics, 79(7), 2064-2072.

Yarmey, A. D. (1991). Descriptions of distinctive and non-distinctive voices over time. Journal of the Forensic Science Society, 31(4), 421-428. 


\section{Supplementary materials}

\section{Section 1: Validating the perceptual properties of the identities}

To better understand the perceptual properties of the identities we created, we ran two pilot studies on the delexicalised stimuli created from Speaker 1. The first pilot consisted of a ratings task, assessing perceived speaker age, speaker sex and naturalness of the four different identities. The second pilot was a speaker discrimination experiment, aiming to validate that the created identities sound sufficiently distinct from each other while also including substantial within-person variability. Since the manipulations of GPR and VTL were similar across the identities created from Speaker 1 and Speaker 2, we only validated the identities created from Speaker 1 and assumed that the identities created from Speaker 2 would behave in similar ways.

\section{How are the identities perceived in terms of sex, age and naturalness?}

We collected ratings for perceived naturalness ("How natural does this sound?" 1-not at all; 7-very much), speaker sex ("How male or female does this sound?" 1-very male; 7-very female), and speaker age ("How much does this sound like an adult?" 1-not at all; 7-very much) based on 7-point Likert scales $(N=15$ per scale; mean age $=30.00$ years, $S D=5.78$ years, 23 female in total). None of the participants was familiar with the original speaker. Ratings were collected online for a subset of locations from each identity ( $N=25$; mapping the full within-person spaces via a regular $5 \times 5$ grid) and based on two sentences produced by Speaker 1. Ratings were similar for stimuli created from the two sentences; we therefore assumed that all sentences are perceptually similar under the manipulations. Ratings of perceived naturalness indicated that one identity (grey in Figure 1, main manuscript: low GPR, short VTL) was perceived to be the least natural-sounding identity $(M=2.42, S D=.43)$. Upon reviewing the stimuli, it was determined that these low ratings were likely the result of manipulation artefacts affecting the stimuli for this identity (buzzlike voice quality). This identity was thus excluded from all further tests. Ratings of speaker sex and speaker age indicated that the remaining 3 identities all sounded broadly male (Means: ID1 =3.47; ID2 = 1.56; ID3 = 3.28) and broadly adult (Means: ID1: 3.82; ID2 = 6.22; ID3 = 4.93). 
Are the identities perceived as distinct from each other, and are the within-person spaces big enough?

We ran a speaker discrimination task online on a subset of items per identity ( 9 items x 3 identities = 27 stimuli), again mapping the full within-person identity spaces via a regular grid. Stimuli were based on the full 69 recorded sentences. Thirty-six participants (mean age $=28.1$ years,$S D=6.4$ years, 19 female) who were not familiar with the original speakers took part in this study. Each participant performed a speaker discrimination task ("Same or different speaker?") on one third of all the possible pairings for the 27 voice space locations (378 pairs per participant), so that each pair was judged by 12 listeners. This pilot study showed that the 3 identities were readily perceived as distinct from one another (see Supplementary Figure 1): listeners perceived $90.5 \%$ of trials that included two nominal identities as featuring different speakers. We furthermore observed that the within-person spaces included considerable variability (e.g. Lavan, Burton, Scott \& McGettigan, 2018 for a review): overall, listeners perceived $68.6 \%$ of all trials including a pair of sounds from the same nominal identity as coming from the same speaker. For some pairs sampled from within one nominal identity, listeners more often perceived items to come from two different speakers.
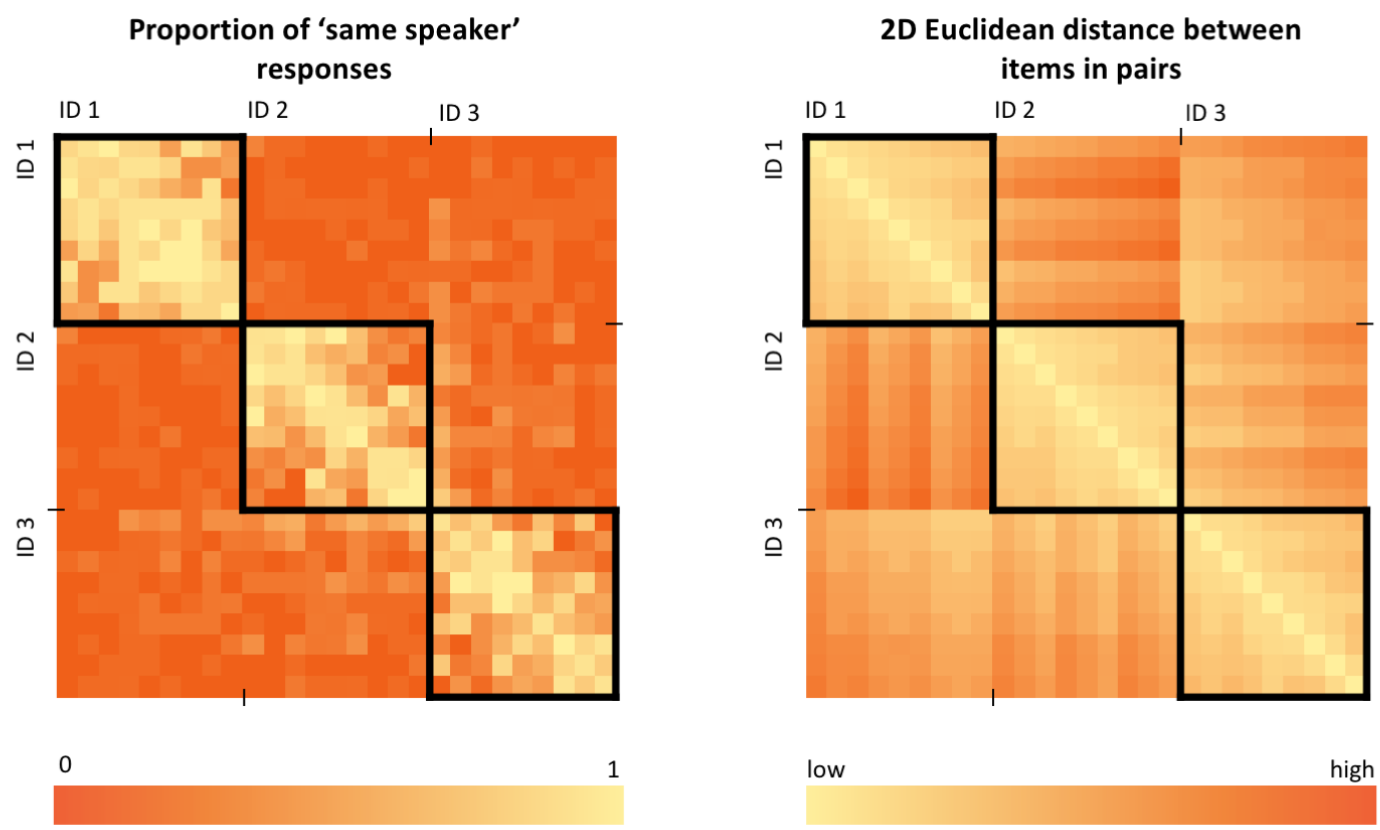

Supplementary Figure 1: Illustration of the results of the speaker discrimination pilot. The matrix on the left shows the pair-wise proportion of 'same speaker' responses, the matrix on the right shows the corresponding pair-wise 2D Euclidean acoustic distance (in GPR x VTL space) between the items within a pair. Within-person submatrices are highlighted with black borders. 


\section{Section 2: Analyses of the distractor identities}

In the main text, we reported exploratory analyses of the data for the distractor trials. We showed that accuracy (i.e. correctly labelling a distractor as "new") was higher for stimuli located on the ring-shaped distribution compared to stimuli located on the centre distribution. Further, accuracy now correspondingly decreased the closer stimuli were to the centre (whether considering both acoustic properties together, or modelling them individually). This is the opposite pattern of results observed for the learned identities.

Before interpreting these results, it is first worthwhile to consider how listeners may have attempted to differentiate between learned and distractor identities: The existing literature on voice discrimination and voice perception has shown that GPR and VTL are used to discriminate between voices (Baumann \& Belin, 2008), although we note that cues used to make identity judgements for voices are likely to be at least partially voice- and listener-specific (e.g. Lavner, Gath \& Rosenhouse, 2000, see also Kreiman \& Sidtis, 2011). In the current experiments, GPR for the learned and distractor identities was matched precisely through manipulation, while identities also substantially overlapped substantially in the VTL dimension. By minimising the differences in GPR and VTL between the learned and distractor identities, these cues were of reduced informative value in discriminating between them. Listeners would thus have needed to use alternative or additional acoustic properties that were not explicitly matched, such as speech rate, F0 variation or periodicity features such as shimmer, jitter or HNR, in order to complete the task within the test phase of the experiments with high accuracy. However, the false alarm rates - where listeners incorrectly identified the distractor identities as "old" - were high across both experiments. This then suggests either that listeners were unsuccessful at making use of additional cues, or that they instead still relied on GPR and VTL despite these properties being largely non-diagnostic in the recognition task

It is unclear why performance differs between the centre and the training distribution for the distractor identities: these were all previously unheard and the distribution of values per se should therefore not have any impact on performance. We can propose a number of speculative explanations for these effects. First, these results may be driven by task-dependent processes: in studies that involve same/different or 
match/mismatch judgements, patterns of results differ according to the trial type (e.g. Narayan, Mak \& Bialystok, 2017; Ritchie \& Burton, 2017; Experiment 3) - this may also be the case for our recognition tests.

We may also speculate that these results could offer additional evidence for the formation of abstracted average representations during learning. First, the distractor identities fully overlap with the GPR range of the learned identities (but note that the VTL range was not fully matched). Due to this overlap, the abstracted average-based representations formed for the learned identities may therefore have provided a good match for the stimuli of the distractor identities, in particular those located in the centre distribution. Thus, distractor items at the centre yielded a greater number of false alarms, i.e. incorrect "old" responses. In contrast, distractor stimuli falling on the ringshaped distribution were further away from the average-based representation, were a worse match to the abstracted average and were thus more readily (and accurately) recognised as a "new" identity. We note, however, that this interpretation of the results is speculative and strongly assumes that only norm-based coding underlies the formation of representations, with exemplar-based representations playing no role. It furthermore assumes ideally overlap between the distractor and learned voices. We know that this was not the case: based on our estimates (see Methods in the main manuscript) distractor and learned identities differed by around $.2 \mathrm{~cm}$ in VTL, which would correspond to a displacement of around 2 manipulation steps in our study. This difference in VTL across speakers may not, however, dramatically affect our interpretation of the results: We note that first, false alarm rates in the recognition task were high, indicating that distractor and learned identities were highly confusable. This confusability thus points at substantial perceptual overlap in perceptual properties, including VTL. Second, the relationship between accuracy and acoustic distance to the centre, while significant, is noisy. Therefore a difference in VTL may only have a limited effect, especially in the presence of matched GPR. Overall, we nonetheless stress that these interpretations of the results of exploratory analyses are highly speculative and should thus be treated accordingly. 


\section{References}

Lavan, N., Burton, A. M., Scott, S. K., \& McGettigan, C. (2018). Flexible voices: Identity perception from variable vocal signals. Psychonomic bulletin \& review, 1-13.

Narayan, C. R., Mak, L., \& Bialystok, E. (2017). Words get in the way: Linguistic effects on talker discrimination. Cognitive science, 41(5), 1361-1376.

Ritchie, K. L., \& Burton, A. M. (2017). Learning faces from variability. Quarterly Journal of Experimental Psychology, 70(5), 897-905. 